\title{
Large-Scale Transposon Mutagenesis of Photosynthetic Bradyrhizobium Sp. Strain ORS278 Reveals New Genetic Loci Putatively Important for Nod-Independent Symbiosis with Aeschynomene indica
}

\author{
Katia Bonaldi, ${ }^{1,2}$ Benjamin Gourion, ${ }^{1}$ Joel Fardoux, ${ }^{1}$ Laure Hannibal, ${ }^{1}$ Fabienne Cartieaux, ${ }^{1}$ \\ Marc Boursot, ${ }^{1}$ David Vallenet, ${ }^{3}$ Clémence Chaintreuil, ${ }^{1}$ Yves Prin, ${ }^{4}$ Nico Nouwen, ${ }^{1}$ and Eric Giraud ${ }^{1}$ \\ ${ }^{1}$ Laboratoire des Symbioses Tropicales et Méditerranéennes, IRD, UMR-IRD/SupAgro/INRA/UM2/CIRAD, F-34398 Montpellier, \\ France; ${ }^{2}$ UM2, F-34398 Montpellier, France; ${ }^{3}$ Genoscope, CNRS-UMR 8030, Atelier de Génomique Comparative, F-91057 \\ Evry, France; ${ }^{4}$ Laboratoire des Symbioses Tropicales et Méditerranéennes, CIRAD, UMR-IRD/SupAgro/INRA/UM2/CIRAD, \\ F-34398 Montpellier, France
}

Submitted 30 October 2009. Accepted 23 February 2010.

\begin{abstract}
Photosynthetic Bradyrhizobium strains possess the unusual ability to form nitrogen-fixing nodules on a specific group of legumes in the absence of Nod factors. To obtain insight into the bacterial genes involved in this Nod-independent symbiosis, we screened 15,648 Tn5 mutants of Bradyrhizobium sp. strain ORS278 for clones affected in root symbiosis with Aeschynomene indica. From the 268 isolated mutants, 120 mutants were altered in nodule development $\left(\mathrm{Ndv}^{-}\right)$and 148 mutants were found to be deficient in nitrogen fixation $\left(\mathrm{Fix}^{-}\right)$. More than $50 \%$ of the $\mathrm{Ndv}^{-}$mutants were found to be altered in purine biosynthesis, strengthening the previous hypothesis of a symbiotic role of a bacterial purine derivative during the Nod-independent symbiosis. The other $\mathrm{Ndv}^{-}$mutants were auxotrophic for pyrimidines and amino acids (leucine, glutamate, and lysine) or impaired in genes encoding proteins of unknown function. The $\mathrm{Fix}^{-}$mutants were found to be affected in a wide variety of cellular processes, including both novel $(n=56)$ and previously identified $(n=31)$ genes important in symbiosis. Among the novel genes identified, several were involved in the Calvin cycle, suggesting that $\mathrm{CO}_{2}$ fixation could play an important role during this symbiosis.
\end{abstract}

Nitrogen-fixing symbiosis between rhizobia and legume plants plays an important role in agriculture and natural ecosystems. This interaction typically results in the formation of a specialized root organ, the nodule, which houses the bacteria. The initiation and development of nodules are mediated by an exchange of diffusible signal molecules between both partners that controls the specificity of recognition, the nodule organogenesis, and the infection process (Perret et al. 2000; Jones et al. 2007; Gibson et al. 2008). Initially, flavonoids exuded by the host plant interact with rhizobial NodD regulatory proteins and induce the expression of nodulation genes (nod, nol, and noe). This leads to the synthesis and secretion of rhizobial lipochito-oligosaccharide signal molecules called Nod factors

Corresponding author: Eric Giraud; Telephone: 33 467593783; Fax: 33 467593802; E-mail: eric.giraud@ird.fr

*The $\boldsymbol{e}$-Xtra logo stands for "electronic extra" and indicates that Figures 1 and 2 appear in color online.
(NF). The perception of NF by plant kinases containing LysM domains triggers, in turn, a number of early responses in the plant root (ion fluxes, rhythmic calcium oscillations, root hair curling, and cortical cell divisions) that lead to the formation of a nodule primordium and the penetration of bacteria (Geurts et al. 2005; Stacey et al. 2006; Oldroyd and Downie 2008). In general, rhizobia penetrate the plant via a sophisticated infection process involving root hair curling and the formation of a host-derived infection thread that guides the bacteria to the emerging nodule primordium. After release from the infection thread, the rhizobia invade the plant cells, differentiate into bacteroids, and reduce atmospheric nitrogen to ammonia to the benefit of the plant. In exchange, the plant supplies the rhizobia with carbon sources.

Three nod genes are essential for the synthesis of the NF core structure: i) nodC encodes an $\mathrm{N}$-acetyl-glucosaminyltransferase that polymerizes UDP-N-acetyl-D-glucosamine into oligosaccharide chains, ii) $\operatorname{nodB}$ encodes a deacetylase that removes the $N$-acetyl moiety from the nonreducing terminus of these oligosaccharides, and iii) nodA encodes an acyltransferase that links an acyl chain to the nonreducing end of the oligosaccharides. In addition to these three canonical nodABC genes, each rhizobium species contains several additional nodulation genes (nod, nol, or noe) encoding proteins that modify the basic NF core structure and that are major determinants of the bacterium host specificity. The canonical nod $A B C$ genes were identified in all characterized rhizobia, including the $\beta$-proteobacteria that nodulate legumes (Moulin et al. 2001; Amadou et al. 2008); therefore, it was supposed that NF were absolutely required for the nodule development in legumes. However, it has been recently shown that the genome of two photosynthetic Bradyrhizobium strains (BTAi1 and ORS278) that elicit nitrogen-fixing nodules on roots and stems of some legumes belonging to the Aeschynomene genus do not contain the nod $A B C$ genes (Giraud et al. 2007). This finding overturned the universality of the NF paradigm and indicated that some rhizobia use an alternative Nod-independent process to establish a symbiotic interaction with a legume plant.

Photosynthetic bradyrhizobia enter Aeschynomene spp. via cracks in the epidermis that result from lateral or adventitious root protrusion as described for the symbiotic couple Sesbania rostrata/Azorhizobium caulinodans (Goormachtig et al. 2004). The formation of infection threads is required to guide Azo- 
rhizobium caulinodans whereas it is assumed that photosynthetic bradyrhizobia progress intercellularly (Arora 1954). This mode of invasion by "crack-entry" in the absence of infection threads has been well described in Arachis hypogea (Chandler 1978) or Stylosanthes spp. (Chandler et al. 1982) and is likely to be common among legumes belonging to the Dalbergieae and Genisteae tribes (Sprent 2007).

In contrast to the well-characterized NF-dependent symbiosis, little is known about the Nod-independent process. In a previous study, 9,600 Tn5 mutants of Bradyrhizobium sp. strain ORS278 were screened for clones affected in the induction of nodules on the roots of Aeschynomene sensitiva (Giraud et al. 2007). However, none of the selected mutants was found to be completely deficient in nodulation. Because Bradyrhizobium sp. strain ORS278 has a large genome (7.5 $\mathrm{Mb})$, the number of tested mutants might not have been sufficient to cover the whole genome, and essential genes for the Nod-independent nodule organogenesis could have been missed. In addition, during this previous study, mutants that formed nodules with a normal shape but that were nitrogen deficient $\left(\mathrm{Fix}^{-}\right)$were not considered whereas the later steps of this new symbiotic system could also differ.

This study had two goals: first, extend the knowledge of the bacterial genes involved during the early steps of this Nodindependent symbiosis and, second, identify the bacterial genes involved during the later steps in order to understand the physiology of the bacteria in this new symbiotic context. For this purpose, we screened 15,648 additional Tn5 mutants of strain ORS278 for clones deficient in their ability to induce nodules or to fix nitrogen. With this genome-wide mutational analysis, we were able to identify important metabolite routes and regulatory circuits employed by photosynthetic bradyrhizobia during symbiosis with Aeschynomene spp.

\section{RESULTS}

\section{Isolation of $\operatorname{Tn} 5$ mutants}

of Bradyrhizobium sp. strain ORS278 affected in symbiosis.

To isolate new bacterial genes involved in the Nod-independent nodulation process, a large-scale screening was carried out using 15,648 additional Tn5 mutants of Bradyrhizobium sp. strain ORS278. This screening was basically performed in 48 wells plates as described by Giraud and associates (2007) but, because of a lack of seed availability and handling facilities, $A$. indica, a species of the same cross inoculation group as A. sensitiva, was used. In this plant culture system, $A$. indica inoculated with the wild-type (WT) strain displayed first nodules after 1 week and the root system harbored 5 to 10 nodules after 3 weeks. The nodules were always located at the emergences of lateral roots; they displayed a spherical shape with a diameter of 1 to $2 \mathrm{~mm}$ and exhibited a dark-green color due to the formation of chloroplasts in the nodule parenchyma and cortex upon light exposure of the root system (Fig. 1A). In the first round of screening, 1,992 mutants eliciting nodules with an unusual phenotype in shape, size, or color were selected, of which 268 could be confirmed (Fig. 1A). Among them, 120 were altered in their ability to induce nodules with a normal shape and were termed nodule-development-defective $\left(\mathrm{Ndv}^{-}\right)$ mutants. However, no completely deficient mutant $\left(\mathrm{Nod}^{-}\right)$ could be found because the formation of bumps was observed on a small number of plants, even for the most affected mutants. The 148 other mutants elicited WT-shaped nodules with a yellow color indicative of nitrogen fixation deficiency (Noel et al. 1988) and were termed Fix ${ }^{-}$. Phenotypic characteristics (symbiotic property, growth rate, and nitrogenase activity) of the selected $\mathrm{Ndv}^{-}$and $\mathrm{Fix}^{-}$mutants are displayed in Tables 1 and 2, respectively. For each mutant, additional data, including pictures of the corresponding nodules and the genomic context of the altered genes, can be found on online (GenoScope MaGe NewNodBrady database). This web resource is integrated into the Rhizoscope MaGe project that offers many connections to other databases or systems, especially the metabolic or genomic databases built for Rhizobium spp. (Vallenet et al. 2006).

A significant variation in the severity of the nodulation deficiency was observed in the $\mathrm{Ndv}^{-}$class, ranging from mutants that only induced bumps or very rare pseudonodules on a small fraction of the plants to mutants that induced protuberant pseudonodules on all the plant tested (Fig. 1). Examination of bacterial growth on rich and minimal media indicated that the $\mathrm{Ndv}^{-}$mutants were affected in their general metabolism, except for the mutants in BRADO0213, BRADO4535, and BRADO6039 (Table 1). As expected, none of the plants inoculated with $\mathrm{Ndv}^{-}$mutants fixed nitrogen.

Within the class of $\mathrm{Fix}^{-}$mutants, two types could be distinguished. Type 1 mutants induced only yellow nodules whereas type 2 mutants induced light-green nodules or a mixture of yellow and light-green nodules on the same plants (Fig. 1A). Measurements of nitrogenase activity confirmed that all the mutants initially selected on the basis of a difference in nodule color were actually affected in their ability to fix nitrogen in symbiosis with $A$. indica and, among them, the type I mutants were affected most drastically.

\section{Determination of $\mathbf{T n} 5$ insertion sites.}

Tn5 insertion sites in the selected mutants were mapped using a two-stage semidegenerate polymerase chain reaction (PCR) and sequencing of the $5^{\prime}$ transposon boundary (Jacobs et al 2003). This approach was successful for $91 \%$ of the tested mutants. Although all clones corresponded to different insertion events, for some coding sequences (CDS), multiple Tn5 insertions were found (up to 19 insertions). For the $120 \mathrm{Ndv}^{-}$ mutants, the Tn5 was inserted into 29 different CDS and, for the $148 \mathrm{Fix}^{-}$mutants, the Tn5 insertion affected 87 different CDS (Tables 1 and 2). These mutants were classified according to the metabolic pathways or cellular processes that were altered. It appeared that $\mathrm{Ndv}^{-}$mutants were mainly affected in the purine and leucine biosynthesis pathways whereas Fix mutants were affected in a wide variety of cellular processes (nitrogenase activity, energetic and carbon metabolism, gluconeogenesis, transport, $\mathrm{CO}_{2}$ fixation, regulation, chemotaxis, and so on).

In rhizobial genomes, symbiosis-specific genes are usually clustered on plasmids or in chromosomal genomic regions acquired by horizontal transfer and characterized by a low GC content (Barnett et al. 2001; Kaneko et al. 2002; Young et al. 2006). Mapping of the Tn5 insertions of $\mathrm{Ndv}^{-}$and $\mathrm{Fix}^{-} \mathrm{mu}-$ tants on the Bradyrhizobium ORS278 genome did not permit identification of a clear hot spot integration site in such regions (Fig. 1B), suggesting the absence of a genomic region specifically dedicated to the symbiotic process. Similarly, no clear region could be identified by omitting the mutants affected in central metabolism in this mapping (data not shown).

\section{DISCUSSION}

The largest genetic screen in the rhizobia/legumes field.

In this study, to better understand the molecular basis of the Nod-independent symbiosis between photosynthetic bradyrhizobia and Aeschynomene spp. we screened 15,648 Tn5 mutants of strain ORS278 for their inability to induce nodules or to fix nitrogen in association with plants. Considering the 9,600 mutants previously tested on A. sensitiva, 25,248 mutants, in total, were analyzed, making this the most extensive 
A
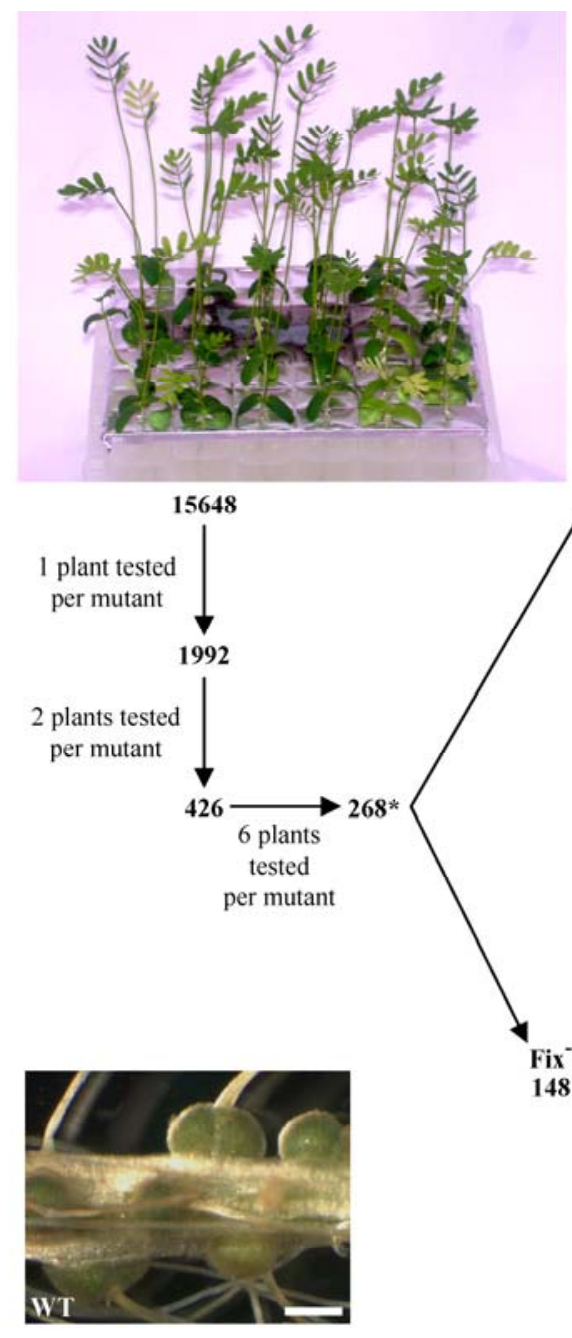
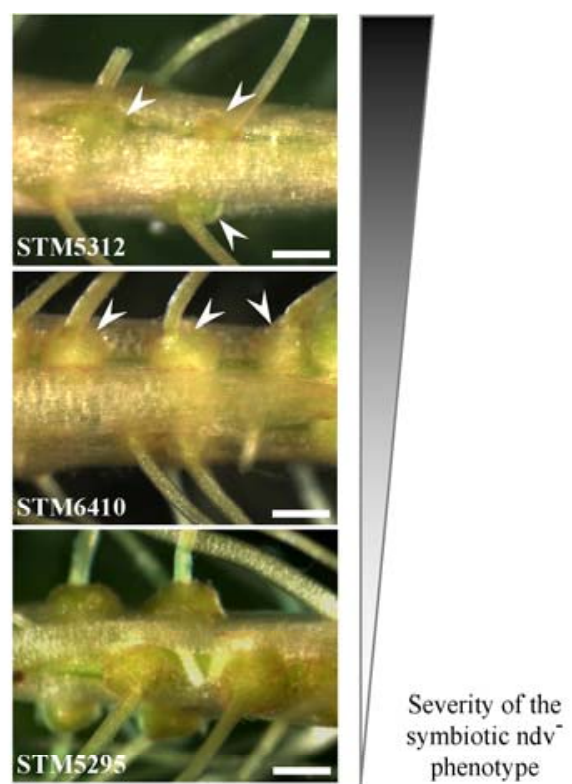

symbiotic ndv ${ }^{-}$ phenotype

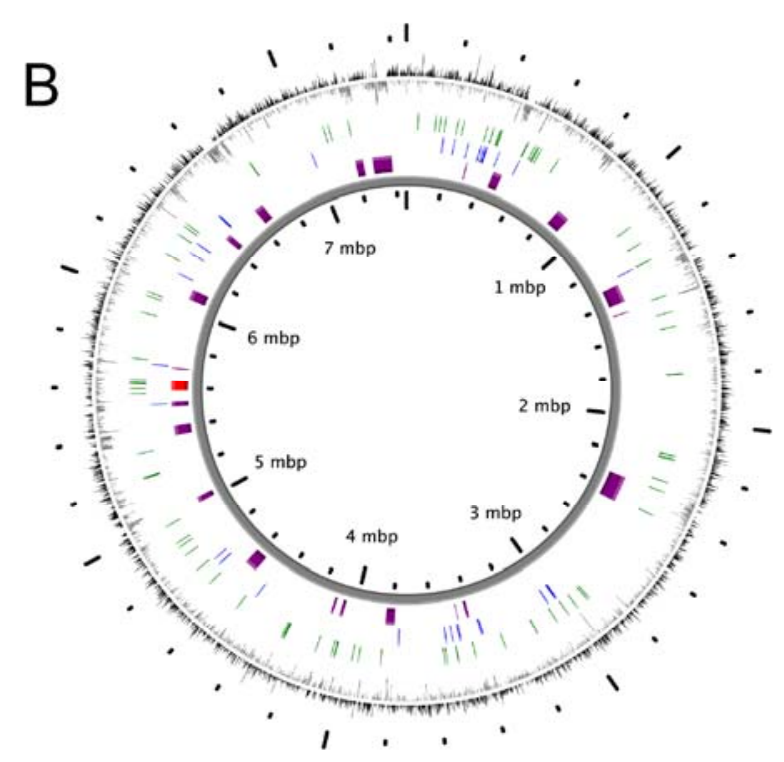

Fig. 1. Screening of the Tn5 mutant library. A, Screening strategy used to select symbiotically deficient clones. The plant culture system in microplate, the typical appearance of nodules elicited by the wild-type (WT) strain, and nodule development-defective $\left(\mathrm{Ndv}^{-}\right)$and nitrogen-deficient $\left(\right.$Fix $\left.{ }^{-}\right)$mutants are shown. Scale bars $=1 \mathrm{~mm}$. White arrowheads show bumps or pseudonodules elicited by the $\mathrm{Ndv}^{-}$mutants. The final number of mutants selected corresponds to the mutants for which the symbiotic deficiency was confirmed for all the plants tested and for which the Tn5 insertion site was mapped. B, Genomic localization of the Tn5 insertions of the selected clones. Circles displayed from the outside show i) GC percent deviation (GC window - mean GC) in a 1,000-bp window, ii) $\mathrm{Fix}^{-}$transposon insertions in genes (green), iii) $\mathrm{Ndv}^{-}$transposon insertions in genes (blue), and iv) putative horizontally acquired genomic island (purple) and the nif/fix region (red) (Giraud et al. 2007). 
screening of a Tn5 mutant library reported to date for rhizobia. Below, genes identified during our study will be discussed. It is important to keep in mind that they remain candidate genes because the symbiotic defect of the corresponding mutants is not necessarily caused by the observed $\operatorname{Tn} 5$ insertion but could result from polar effect or from a secondary unidentified mutation. Therefore, further investigations are necessary to confirm their symbiotic role.

Absence of a complete Nod $^{-}$mutant in the Tn5 collection.

In all, 120 mutants altered in 29 different CDS were identified as severely impaired in nodulation. Surprisingly, none of them was completely nodulation deficient. This might be due to a high degree of redundancy in the nodulation determinants, either through the presence of homologous genes (more than $20 \%$ of ORS278 genes have paralogues) or of different genes that are functionally interchangeable. Alternatively, it is possible that nodule organogenesis in A. indica is not triggered by a specific signal as NF but by a synergistic action of multiple factors that could partially complement each other. Finally, two other hypotheses explaining the absence of the isolation of a strict $\mathrm{Nod}^{-}$mutant should be considered: i) the genes necessary for symbiosis are essential for bacterial survival and ii) key genes necessary to induce nodule organogenesis have been missed in our screen. Although our mutant collection provides almost $4 \times$ coverage of all predicted CDS $(6,752)$, we cannot completely exclude the latter hypothesis because a nonrandom distribution of the Tn5 insertions is likely and the chance to disrupt small genes is low (Boyd et al. 1993; Jacobs et al. 2003).

Most of the $\mathrm{Ndv}^{-}$mutants were auxotrophic for nucleotides (purines or pyrimidines) or amino acids (Glu, Leu, and Lys). Two classes of auxotrophs emerged, purine and leucine mutants that represented 54 and $29 \%$ of the $\mathrm{Ndv}^{-}$mutants, respectively. Purine and leucine auxotrophs were among the first mu-

Table 1. Phenotypes and $\operatorname{Tn} 5$ insertion sites of nodule development-deficient mutants

\begin{tabular}{|c|c|c|c|c|c|c|}
\hline \multirow[b]{2}{*}{ Functional class, CDS $^{\mathbf{b}}$} & \multirow[b]{2}{*}{ Collection $^{c}$} & \multirow[b]{2}{*}{ Gene annotation $^{\mathrm{d}}$} & \multirow[b]{2}{*}{ Mutant no. ${ }^{\mathrm{e}}$} & \multicolumn{2}{|c|}{ Growth rate $(\%)^{\mathrm{a}}$} & \multirow[b]{2}{*}{$\mathbf{N}_{2}$ rate $^{f}$} \\
\hline & & & & YMm & MM & \\
\hline Nucleotides biosynthesis & $\ldots$ & $\ldots$ & 64 & $\ldots$ & $\ldots$ & $\ldots$ \\
\hline Purines & $\ldots$ & $\ldots$ & 58 & $\ldots$ & $\ldots$ & $\ldots$ \\
\hline BRADO0338 & STM6333 & purH & 14 & 65 & 10 & ND \\
\hline BRADO1206 & STM6338 & purE & 3 & 93 & 13 & ND \\
\hline BRADO2779 & STM5423 & purQ & 1 & 83 & 12 & ND \\
\hline BRADO2785 & STM6410 & pur $\widetilde{L}$ & 10 & 85 & 12 & ND \\
\hline BRADO2830 & STM5308 & purB & 14 & 94 & 4 & ND \\
\hline BRADO3165 & STM5295 & gиав & 7 & 54 & 12 & ND \\
\hline BRADO3169 & STM5326 & guaA & 2 & 51 & 14 & ND \\
\hline BRADO3280 & STM5291 & purF & 1 & 91 & 14 & ND \\
\hline BRADO3344 & STM5402 & purM & 1 & 86 & 13 & ND \\
\hline BRADO3345 & STM6326 & purN & 2 & 92 & 11 & ND \\
\hline BRADO6088 & STM6408 & purD & 3 & 95 & 11 & ND \\
\hline Pyrimidines & $\ldots$ & $\ldots$ & 6 & $\ldots$ & $\ldots$ & $\ldots$ \\
\hline BRADO4502 & STM6358 & pyrB & 1 & 63 & 6 & ND \\
\hline BRADO4503 & STM6396 & pyrC & 4 & 59 & 6 & ND \\
\hline BRADO6742 & STM5443 & pyrD & 1 & 52 & 4 & ND \\
\hline Amino acids biosynthesis & $\ldots$ & $\ldots$ & 45 & $\ldots$ & $\ldots$ & $\ldots$ \\
\hline Leucine & $\ldots$ & $\ldots$ & 38 & $\ldots$ & $\ldots$ & $\ldots$ \\
\hline BRADO0353 & STM5281 & leuB & 2 & 68 & 11 & ND \\
\hline BRADO0365 & STM5333 & leuD & 7 & 88 & 7 & ND \\
\hline BRADO0374 & STM5264 & leuC & 19 & 66 & 8 & ND \\
\hline BRAD05528 & STM5266 & leuA & 6 & 63 & 5 & ND \\
\hline BRADO6039 & STM5360 & put. IlvE-like protein & 1 & 86 & 70 & ND \\
\hline Glutamate & & $\ldots$ & 8 & $\ldots$ & $\ldots$ & \\
\hline BRADO6238 & STM5257 & gltB & 7 & 91 & $3^{*}$ & ND \\
\hline BRAD06240 & STM5258 & gltD & 1 & 95 & $6^{*}$ & ND \\
\hline Lysine & $\ldots$ & $\ldots$ & 2 & $\ldots$ & $\ldots$ & $\ldots$ \\
\hline BRADO0158 & STM5328 & $d a p B$ & 2 & 53 & 54 & ND \\
\hline Others & $\ldots$ & $\ldots$ & 3 & $\ldots$ & $\ldots$ & $\ldots$ \\
\hline BRADO0277 $^{\mathrm{g}}$ & STM6339 & folD & 1 & 74 & 12 & ND \\
\hline BRADO3272 $^{\mathrm{h}}$ & STM6376 & put. transcriptional regulatory protein TetR/AcrR family & 1 & 92 & 13 & ND \\
\hline BRADO4272 ${ }^{\mathrm{i}}$ & STM6378 & ssuA & 1 & 89 & 12 & ND \\
\hline Proteins of unknown function & $\ldots$ & $\ldots$ & 8 & $\ldots$ & $\ldots$ & $\ldots$ \\
\hline BRADO0213 & STM5312 & CHP & 4 & 100 & 78 & ND \\
\hline BRADO2778 & STM6304 & CHP & 2 & 92 & 38 & ND \\
\hline BRAD04535 & STM5302 & CHP & 1 & 86 & 95 & ND \\
\hline BRADO5309 & STM6553 & $\mathrm{CHP}$ & 1 & 78 & 10 & ND \\
\hline
\end{tabular}

${ }^{a}$ Relative growth rate (percentage of wild type [WT]) estimated on rich medium (yeast extract mannitol [YMm]) or minimal medium (MM); * indicates relative growth rate (percentage of WT) estimated on MM supplemented by $15 \mathrm{mM} \mathrm{KNO}_{3}$ instead of glutamate.

${ }^{\mathrm{b}} \mathrm{CDS}$ names are indicated according to the published genome sequence (Giraud et al. 2007). Coding sequences (CDS) in bold correspond to genes that have not been isolated during the first screening performed on Aeschynomene sensitiva and CDS in italic correspond to mutants for which the Tn5 insertion is located upstream of the CDS.

${ }^{\mathrm{c}}$ Representative mutant strain used to estimate growth and nitrogen fixation rate.

${ }^{\mathrm{d}}$ Abbreviations: put., putative; CHP, conserved hypothetical protein.

${ }^{\mathrm{e}}$ Number of independent mutants identified in the corresponding CDS.

${ }^{\mathrm{f}}$ Fixation rate: relative acetylene reduction activity estimated on whole plants; ND = not detected (percentage of WT).

g 10-Formyl tetrahydrofolate biosynthesis.

${ }^{\text {h }}$ Specific to photosynthetic Bradyrhizobium.

${ }^{\mathrm{i}}$ Sulfonate $\mathrm{ABC}$ transporter.

${ }^{\mathrm{j}}$ Upstream of $\operatorname{pur} Q$. 
tants described as $\mathrm{Ndv}^{-}$in previous genetic screens on classical rhizobium (Dénarié and Bergeron 1976; Pain 1979; Long 1989). It is still not clear whether their phenotype is due to growth impairment or to an alteration of the symbiotic process. It has been proposed that a purine precursor (AICAR) could play a symbiotic role (Noel et al. 1988; Newman et al. 1994); however, the selection of several mutants altered in genes involved in downstream steps of the purine pathway suggests that AICAR does not play such a role in the Nod-independent symbiosis. The production by rhizobia of a cytokinin-like compound from adenine was also proposed to stimulate host cell division (Pain 1979). This old hypothesis, which had been ruled out with the discovery of Nod factors, resurfaces today with the finding that a Lotus japonicus gain-of-function mutant in a cytokinin receptor (LHK1) develops nodules spontaneously in the absence of rhizobia (Tirichine et al. 2007). It has

Table 2. Phenotypes and Tn5 insertion sites of nitrogen deficient $\left(\mathrm{Fix}^{-}\right)$mutants

\begin{tabular}{|c|c|c|c|c|c|c|c|c|}
\hline \multirow[b]{2}{*}{ Functional class, $\operatorname{CDS}^{\mathrm{b}}$} & \multirow[b]{2}{*}{ Collection $^{\mathrm{c}}$} & \multirow[b]{2}{*}{ Gene annotation ${ }^{\mathrm{d}}$} & \multirow[b]{2}{*}{ Mutant $^{\mathrm{e}}$} & \multicolumn{2}{|c|}{ Growth rate $(\%)^{\mathrm{a}}$} & \multirow[b]{2}{*}{$\mathrm{N}_{2}(\%)^{\mathrm{f}}$} & \multirow[b]{2}{*}{ Fix $^{-g}$} & \multirow[b]{2}{*}{ Comments } \\
\hline & & & & YMm & MM & & & \\
\hline Nitrogenase activity & $\ldots$ & $\ldots$ & 6 & $\ldots$ & $\ldots$ & $\ldots$ & $\ldots$ & $\ldots$ \\
\hline BRADO5394 & STM6439 & nifH & 1 & 95 & 115 & 80 & $\mathrm{t} 2$ & $\ldots$ \\
\hline BRADO5436 & STM6466 & nifN & 2 & 100 & 121 & ND & $\mathrm{t} 1$ & $\ldots$ \\
\hline BRADO5437 & STM6469 & nifE & 1 & 100 & 120 & ND & $\mathrm{t} 1$ & $\ldots$ \\
\hline BRADO5438 & STM6453 & nifK & 2 & 79 & 128 & ND & $\mathrm{t} 1$ & $\ldots$ \\
\hline Nif genes regulators & $\ldots$ & $\ldots$ & 16 & $\ldots$ & $\ldots$ & $\ldots$ & $\ldots$ & $\ldots$ \\
\hline BRADO0113 & STM6420 & rpoN & 1 & 60 & 119 & ND & $\mathrm{t} 1$ & $\ldots$ \\
\hline BRADO0530 & STM6479 & $\operatorname{gln} D$ & 9 & 91 & 74 & 42 & $\mathrm{t} 2$ & $\ldots$ \\
\hline BRADO3866 & STM6448 & $n t r C$ & 1 & 98 & 102 & ND & $\mathrm{t} 1$ & $\ldots$ \\
\hline BRADO3867 & STM6309 & $n t r B$ & 2 & 109 & 108 & ND & $\mathrm{t} 1$ & $\ldots$ \\
\hline BRADO3868 & STM6409 & put. NifR3-like protein & 1 & 93 & 38 & ND & $\mathrm{t} 1$ & $\ldots$ \\
\hline BRADO5449 & STM5456 & nifA & 2 & 102 & 95 & ND & $\mathrm{t} 1$ & $\ldots$ \\
\hline Electron transfers & $\ldots$ & $\ldots$ & 10 & $\ldots$ & $\ldots$ & $\ldots$ & $\ldots$ & $\ldots$ \\
\hline \multirow[t]{2}{*}{ BRADO2020 } & STM6351 & put. electron transfer & & & & & & \\
\hline & & flavoprotein dehydrogenase & 5 & 54 & 62 & 19 & $\mathrm{t} 2$ & $\ldots$ \\
\hline BRADO2734 & STM6375 & cycK & 1 & 76 & 76 & 27 & $\mathrm{t} 2$ & $\ldots$ \\
\hline \multirow[t]{2}{*}{ BRADO4317 } & STM6330 & put. pyruvate ferredoxin/ & & & & & & \\
\hline & & flavodoxin oxidoreductase & 1 & 84 & 106 & ND & $\mathrm{t} 1$ & $\ldots$ \\
\hline BRAD06491 & STM5341 & etfa & 2 & 45 & 45 & 24 & $\mathrm{t} 2$ & $\ldots$ \\
\hline BRADO6492 & STM5415 & etfB & 1 & 44 & 47 & 26 & $\mathrm{t} 2$ & $\ldots$ \\
\hline Central metabolism & $\ldots$ & $\ldots$ & 37 & $\ldots$ & $\ldots$ & . & $\ldots$ & $\ldots$ \\
\hline BRADO0157 & STM6303 & gртA & 1 & 80 & 31 & 5 & $\mathrm{t} 1$ & $\ldots$ \\
\hline BRADO0234 & STM6321 & $s d h A$ & 1 & 82 & 43 & ND & $\mathrm{t} 1$ & $\ldots$ \\
\hline BRADO0404 & STM6313 & sucC & 3 & 32 & 24 & ND & $\mathrm{t} 1$ & $\ldots$ \\
\hline BRADO0405 & STM6319 & sucD & 4 & 32 & 25 & ND & $\mathrm{t} 1$ & $\cdots$ \\
\hline BRADO0409 & STM6359 & $\operatorname{lpD}$ & 5 & 79 & 61 & 85 & $\mathrm{t} 2$ & $\ldots$ \\
\hline BRADO2034 & STM6550 & $p p d K$ & 1 & 108 & 125 & 34 & $\mathrm{t} 2$ & $\ldots$ \\
\hline BRADO2682 & STM6364 & mutA & 1 & 59 & 119 & 13 & t2 & $\ldots$ \\
\hline BRADO3367 & STM6328 & dme & 1 & 103 & 115 & 86 & $\mathrm{t} 2$ & $\ldots$ \\
\hline BRADO4083 & STM5393 & $\operatorname{lpD}$ & 1 & 86 & 46 & ND & $\mathrm{t} 1$ & $\ldots$ \\
\hline BRADO4086 & STM5303 & $p d h B$ & 6 & 43 & 37 & ND & $\mathrm{t} 1$ & $\ldots$ \\
\hline BRADO4087 & STM5346 & $p d h A$ & 7 & 46 & 32 & 6 & $\mathrm{t} 1$ & $\ldots$ \\
\hline BRADO4094 & STM5353 & eno & 1 & 51 & 57 & ND & $\mathrm{t} 1$ & $\ldots$ \\
\hline BRADO5016 & STM5332 & fumA & 4 & 82 & 115 & 4 & $\mathrm{t} 1$ & $\ldots$ \\
\hline BRADO5815 & STM6317 & put. amylomaltase & 1 & 100 & 134 & 6 & t1 & $\ldots$ \\
\hline Calvin cycle & $\ldots$ & $\ldots$ & 4 & $\ldots$ & $\ldots$ & $\ldots$ & $\ldots$ & $\ldots$ \\
\hline BRADO1122 & STM6457 & $c b b K$ & 1 & 30 & 91 & 80 & $\mathrm{t} 2$ & $\ldots$ \\
\hline BRADO2274 & STM5345 & $c b b L$ & 1 & 78 & 118 & 30 & $\mathrm{t} 2$ & $\ldots$ \\
\hline BRADO2842 & STM5301 & $c b b E$ & 2 & 84 & 13 & ND & $\mathrm{t} 1$ & $\ldots$ \\
\hline Amino acids biosynthesis & $\ldots$ & $\ldots$ & 3 & & & & & $\ldots$ \\
\hline BRADO0212 & STM5385 & hisB & 2 & 40 & 13 & 54 & $\mathrm{t} 2$ & $\ldots$ \\
\hline BRADO5973 & STM6361 & aatA & 1 & 75 & 96 & 50 & $\mathrm{t} 2$ & $\ldots$ \\
\hline Transporters & $\ldots$ & $\ldots$ & 9 & $\ldots$ & $\ldots$ & $\ldots$ & $\ldots$ & $\ldots$ \\
\hline BRADO1675 & STM5361 & $p s t C$ & 2 & 83 & 97 & 3 & $\mathrm{t} 1$ & $\ldots$ \\
\hline BRADO1677 & STM5389 & pstB & 1 & 89 & 107 & ND & $\mathrm{t} 1$ & $\ldots$ \\
\hline BRAD03096 & STM6397 & put. $A B C$ transporter & 1 & 108 & 102 & 22 & $\mathrm{t} 2$ & $\ldots$ \\
\hline BRADO4782 & STM5366 & put. ABC transporter & 1 & 86 & 86 & 5 & $\mathrm{t} 1$ & $\ldots$ \\
\hline BRADO5117 & STM6373 & put. permease & 1 & 94 & 107 & ND & $\mathrm{t} 1$ & $\ldots$ \\
\hline BRADO6119 & STM5363 & put. ABC transporter & 3 & 97 & 106 & 4 & $\mathrm{t} 1$ & $\ldots$ \\
\hline Chemotaxis & $\ldots$ & $\ldots$ & 2 & $\ldots$ & $\ldots$ & $\ldots$ & $\ldots$ & $\ldots$ \\
\hline BRADO1477 & STM5383 & cheR & 1 & 89 & 94 & 3 & $\mathrm{t} 1$ & $\ldots$ \\
\hline \multirow[t]{2}{*}{ BRADO3225 } & STM5381 & put. methyl-accepting & & & & & & \\
\hline & & chemotaxis protein & 1 & 90 & 85 & 11 & $\mathrm{t} 1$ & $\ldots$ \\
\hline
\end{tabular}

${ }^{a}$ Relative growth rate (percentage of wild type [WT]) estimated on rich medium (yeast extract mannitol [YMm) or minimal medium (MM).

${ }^{\mathrm{b}}$ CDS names are indicated according to the published genome sequence (Giraud et al. 2007). CDS in bold correspond to novel symbiotic genes and coding sequences (CDS) in italic correspond to mutants for which the Tn5 insertion is located upstream of the CDS.

${ }^{\mathrm{c}}$ Representative mutant strain used to estimate growth and nitrogen fixation rate.

${ }^{\mathrm{d}}$ Abbreviations : put., putative; HP, hypothetical protein; CHP, conserved hypothetical protein.

${ }^{\mathrm{e}}$ Number of independent mutants identified in the corresponding CDS.

${ }^{\mathrm{f}}$ Fixation rate: relative acetylene reduction activity (percentage of WT) estimated on whole plants; ND = not detected.

${ }^{g}$ Fix $^{-}$phenotype. 
now become clear that cytokinins act downstream of Nod factors to mediate primordium formation (Gonzalez-Rizzo et al. 2006; Murray et al. 2007; Oldroyd 2007; Frugier et al. 2008). In a symbiotic system in which NF are not required, we can ask whether bacterial cytokinins could constitute the first signals triggering nodulation. Experiments at the plant and bacterial levels are currently in progress to test this hypothesis.

Among the 19 new genes identified as potentially involved in nodule development in this study (Table 1), three are of spe- cial interest: BRADO3272, BRADO0213, and BRADO4535. BRADO3272 encodes a transcriptional regulatory protein of the TetR family. Intriguingly, this CDS as well as the two upstream CDS encoding proteins of unknown function appear specific to photosynthetic bradyrhizobia. Nevertheless, the mutation also causes significant growth impairment in minimal medium (MM), which might be responsible for the observed symbiotic deficiency of this mutant. BRADO0213 and BRADO4535 encode proteins of unknown function. In contrast to the other

Table 2. (continued from preceding page)

\begin{tabular}{|c|c|c|c|c|c|c|c|c|}
\hline \multirow[b]{2}{*}{ Functional class, CDS $^{\mathbf{b}}$} & \multirow[b]{2}{*}{ Collection $^{\mathrm{c}}$} & \multirow[b]{2}{*}{ Gene annotation $^{\mathrm{d}}$} & \multirow[b]{2}{*}{ Mutant $^{\mathrm{e}}$} & \multicolumn{2}{|c|}{ Growth rate $(\%)^{\mathrm{a}}$} & \multirow[b]{2}{*}{$\mathrm{N}_{2}(\%)^{\mathrm{f}}$} & \multirow[b]{2}{*}{$\mathrm{Fix}^{-\mathrm{g}}$} & \multirow[b]{2}{*}{ Comments } \\
\hline & & & & YMm & MM & & & \\
\hline Cofactors biosynthesis & & $\ldots$ & 4 & & & & $\ldots$ & $\ldots$ \\
\hline BRADO2036 & STM5364 & $n a d B$ & 1 & 85 & 104 & 59 & $\mathrm{t} 2$ & $\ldots$ \\
\hline BRADO2133 & STM6315 & put. RibBA-like protein & 3 & 84 & 95 & 4 & $\mathrm{t} 1$ & $\ldots$ \\
\hline Nucleotides biosynthesis & $\ldots$ & $\ldots$ & 8 & $\ldots$ & $\ldots$ & $\ldots$ & $\ldots$ & $\ldots$ \\
\hline BRADO1205 & STM6316 & purK & 7 & 94 & 23 & 58 & $\mathrm{t} 2$ & $\ldots$ \\
\hline BRADO2776 & STM6324 & purC & 1 & 90 & 35 & 32 & $\mathrm{t} 2$ & $\ldots$ \\
\hline Others & $\ldots$ & $\ldots$ & 9 & & $\ldots$ & $\ldots$ & $\ldots$ & $\ldots$ \\
\hline BRADO0045 & STM6447 & $\ln t$ & 1 & 50 & 120 & 69 & $\mathrm{t} 2$ & $\ldots$ \\
\hline \multirow[t]{2}{*}{ BRADO0589 } & STM6394 & put. peptidylpropyl & & & & & & \\
\hline & & isomerase & 1 & 102 & 67 & 17 & $\mathrm{t} 2$ & $\ldots$ \\
\hline \multirow[t]{2}{*}{ BRADO2179 } & STM6459 & put. acylCoA & & & & & & \\
\hline & & dehydrogenase & 1 & 87 & 91 & 6 & t1 & $\ldots$ \\
\hline BRADO2938 & STM6437 & put. hydrolase & 1 & 52 & 94 & 18 & $\mathrm{t} 2$ & $\ldots$ \\
\hline \multirow[t]{2}{*}{ BRADO3673 } & STM6418 & put. cold shock DNA & & & & & & \\
\hline & & binding protein & 1 & 65 & 90 & 90 & $\mathrm{t} 2$ & $\ldots$ \\
\hline BRADO3794 & STM6349 & put. sulfite reductase & 1 & 61 & 6 & 74 & t2 & $\ldots$ \\
\hline \multirow[t]{2}{*}{ BRADO4549 } & STM5430 & put. D-alanyl-D-alanine & & & & & & \\
\hline & & carboxypeptidase & 1 & 105 & 114 & 10 & $\mathrm{t} 2$ & \\
\hline BRADO5368 & STM5351 & put. PhnM protein & 1 & 88 & 96 & 3 & $\mathrm{t} 1$ & $\begin{array}{l}\text { Putative metal-dependant } \\
\text { hydrolase }\end{array}$ \\
\hline BRADO6079 & STM5427 & mutL & 1 & 112 & 109 & 26 & $\mathrm{t} 2$ & DNA mismatch repair \\
\hline Various regulators & & $\ldots$ & 6 & & & & $\ldots$ & $\ldots$ \\
\hline BRADO0560 & STM5434 & put. GGDEF/EAL protein & 1 & 98 & 119 & ND & $\mathrm{t} 2$ & $\ldots$ \\
\hline BRADO1049 & STM5387 & put. PhyR-like protein & 1 & 89 & 102 & 75 & $\mathrm{t} 2$ & $\ldots$ \\
\hline BRADO3946 & STM5395 & put. sensor $H K$ & 1 & 111 & 98 & 3 & $\mathrm{t} 1$ & $\ldots$ \\
\hline BRADO4470 & STM6334 & put. LabA-like protein & 1 & 90 & 112 & 3 & $\mathrm{t} 1$ & $\ldots$ \\
\hline \multirow{3}{*}{ BRADO6832 } & STM6398 & $\begin{array}{l}\text { put. transcriptional } \\
\text { regulator }\end{array}$ & 1 & 91 & 103 & ND & t1 & $\ldots$ \\
\hline & STM6461 & two-component system & & & & & & \\
\hline & & regulator & 1 & 96 & 102 & 56 & t2 & $\ldots$ \\
\hline Translational processes & & $\ldots$ & 16 & & $\ldots$ & $\ldots$ & $\ldots$ & $\ldots$ \\
\hline BRADO0134 & STM5347 & $\operatorname{infC}$ & 1 & 67 & 73 & 23 & $\mathrm{t} 2$ & $\begin{array}{l}\text { Translation initiation factor } \\
\text { IF3 }\end{array}$ \\
\hline BRADO0340 & STM6450 & put. RsmB-like protein & 1 & 61 & 110 & 5 & $\mathrm{t} 2$ & 16S RNA methyltransferase \\
\hline BRADO0376 & STM5386 & trmD & 1 & 103 & 108 & 28 & $\mathrm{t} 2$ & tRNA methyltransferase \\
\hline BRADO0377 & STM5399 & $\operatorname{rim} M$ & 1 & 105 & 86 & 30 & $\mathrm{t} 2$ & Ribosome maturation factor \\
\hline BRADO0571 & STM6399 & put. HemK-like protein & 5 & 65 & 103 & 6 & $\mathrm{t} 2$ & $\ldots$ \\
\hline BRADO3306 & STM6363 & 50s ribosomal protein $L 9$ & 2 & 70 & 57 & 75 & $\mathrm{t} 2$ & $\ldots$ \\
\hline BRADO3308 & STM5412 & $r p s R$ & 1 & 99 & 115 & 68 & $\mathrm{t} 2$ & $30 \mathrm{~S}$ ribosomal protein \\
\hline BRADO3359 & STM6485 & aspS & 1 & 88 & 113 & 16 & $\mathrm{t} 2$ & Aspartyl-tRNA synthetase \\
\hline BRADO3881 & STM6480 & vals & 1 & 72 & 117 & 67 & $\mathrm{t} 2$ & Valine-tRNA ligase \\
\hline BRADO6137 & STM5452 & rpmJ & 1 & 84 & 112 & 74 & $\mathrm{t} 2$ & 50S ribosomal protein \\
\hline Proteins of unknown function & & $\ldots$ & 19 & 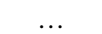 & 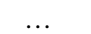 & $\ldots$ & $\ldots$ & $\ldots$ \\
\hline BRADO0575 & STM5349 & $\mathrm{CHP}$ & 2 & 91 & 85 & ND & $\mathrm{t} 1$ & $\ldots$ \\
\hline BRADO0665 & STM6381 & $H P$ & 1 & 82 & 93 & 76 & $\mathrm{t} 2$ & $\begin{array}{l}\text { Specific to photosynthetic } \\
\text { Bradyrhizobium }\end{array}$ \\
\hline BRAD01364 & STM6464 & CHP & 1 & 31 & 98 & 37 & $\mathrm{t} 2$ & $\ldots$ \\
\hline BRADO1426 & STM6393 & $H P$ & 1 & 118 & 18 & 4 & $\mathrm{t} 1$ & $\begin{array}{l}\text { Specific to photosynthetic } \\
\text { Bradyrhizobium }\end{array}$ \\
\hline BRADO2671 & STM6320 & $\mathrm{CHP}$ & 2 & 27 & 51 & 55 & $\mathrm{t} 2$ & $\ldots$ \\
\hline BRADO2937 & STM6314 & $\mathrm{CHP}$ & 1 & 97 & 20 & 56 & t2 & $\ldots$ \\
\hline BRADO3770 & STM5331 & $\mathrm{CHP}$ & 2 & 97 & 95 & ND & $\mathrm{t} 1$ & $\ldots$ \\
\hline BRADO4085 & STM5344 & $\mathrm{CHP}$ & 1 & 52 & 40 & ND & $\mathrm{t} 1$ & $\ldots$ \\
\hline BRADO4683 & STM6438 & $\mathrm{CHP}$ & 1 & 45 & 119 & 64 & $\mathrm{t} 2$ & $\ldots$ \\
\hline BRADO4701 & STM6452 & $\mathrm{CHP}$ & 1 & 60 & 116 & 10 & $\mathrm{t} 2$ & $\ldots$ \\
\hline BRADO5022 & STM5356 & $\mathrm{CHP}$ & 1 & 92 & 76 & 6 & $\mathrm{t} 2$ & $\ldots$ \\
\hline BRAD05546 & STM6386 & CHP & 1 & 109 & 83 & 6 & $\mathrm{t} 1$ & $\ldots$ \\
\hline BRADO5734 & STM6360 & CHP & 1 & 83 & 15 & ND & $\mathrm{t} 1$ & $\ldots$ \\
\hline BRAD05803 & STM5377 & $H P$ & 1 & 92 & 97 & ND & $\mathrm{t} 1$ & $\ldots$ \\
\hline BRAD06852 & STM5340 & CHP & 1 & 86 & 114 & ND & $\mathrm{t} 1$ & $\ldots$ \\
\hline BRAD06923 & STM6417 & $H P$ & 1 & 90 & 80 & 11 & $\mathrm{t} 1$ & $\ldots$ \\
\hline
\end{tabular}


$\mathrm{Ndv}^{-}$mutants, their growth in MM medium was hardly affected, suggesting that their symbiotic phenotype results from a direct modification of the symbiotic process. However, Bradyrhizobium japonicum USDA110 has homologues of both CDS in a genomic context similar to that found in the ORS278 genome. Therefore, it seems unlikely that BRADO0213 and BRADO4535 are specific determinants of the Nod-independent symbiosis.

\section{Novel insights in bradyrhizobial physiology from known nitrogen fixation-related genes.}

To get insights into the bacterial physiology during symbiosis, mutants impaired in nitrogen fixation were selected. Among them were 74 mutants altered in 31 genes known to be involved in bacteroid functioning (Table 2; Fig. 2). These genes participate in i) nitrogenase complex formation (nifK, nifE, nifN, and nifH), ii) nif genes regulation (nifA, rpoN, glnD, $n \operatorname{tr} C$, ntrB, and nifR3), iii) tricarboxylic acid cycle (TCA) (sdhA, sucC, sucB, lpD, fumA, pdhA, pdhB, and dme), iv) gluconeogenesis ( $p p d K$, gpm $A$, and eno), v) transport (pst $B$ and pst $C$ ), vi) amino-acid biosynthesis (hisB and aatA), and vii) in the general stress response recently described in $B$. japonicum (phyR) (Gourion et al. 2009).

Although these genes were already reported to play a role in symbiosis, new insights into the physiology of photosynthetic bradyrhizobia emerged.

i) In contrast to B. japonicum and Sinorhizobium meliloti, mutants in the two-component system NtrB/NtrC were drastically impaired in nitrogen fixation (Szeto et al. 1987; Martin et al. 1988). Regulation of nif genes in rhizobia depends directly on the NifA protein, expression of which is under the control of additional regulatory circuits that differ markedly between Rhizobium spp. (Fischer 1994). Therefore, it is possible that, in photosynthetic bradyrhizobia, nifA is under the control of the nitrogen regulatory (ntr) system.

ii) We isolated several mutants in three key gluconeogenic enzymes: enolase (Eno), phosphoglyceromutase (GpmA), and pyruvate phosphate dikinase (PPDK). In contrast to eno and gpmA mutants, which were completely deficient for nitrogen fixation, the $p p d K$ mutant retained $34 \%$ of the WT nitrogenase activity. The partial activity of the latter most likely results from the presence of an alternative route for phosphoenol pyruvate (PEP) formation involving the PEP carboxykinase (PCK) (Fig. 2).

iii) We finally isolated two $\mathrm{Fix}^{-}$mutants putatively involved in the degradation of the major storage compounds commonly found in rhizobia, poly- $\beta$-hydroxybutyrate (PHB) and glycogen (Lodwig et al. 2005; Trainer and Charles 2006). BRADO2682 encodes the methylmalonyl-CoA mutase (MutA), an enzymatic activity that is required for PHB degradation but which is dispensable during symbiosis in $S$. meliloti (Charles and Aneja 1999). BRADO5815 encodes 4- $\alpha$-glucanotransferase (amylomaltase), implicated in glycogen catabolism. It has been suggested that PHB and glycogen accumulated by the bacteria prior to infection serve to fuel bacteroid formation (Lodwig et al. 2005). Therefore, it is possible that the weak nitrogenase activity measured for both mutants (13 and $6 \%$, respectively) results from an alteration in bacteroid formation. Accordingly, preliminary microscopic observations revealed that, in mature nodules elicited by both mutants, bacteria were undifferentiated (data not shown).

\section{The Fix $^{-}$mutants reveal novel symbiotic genes required for nitrogen fixation.}

In addition to known symbiotic genes, we identified 56 new genes potentially involved in symbiotic nitrogen fixation. Some of these genes are grouped by physiological function and are described below.

A putative role for $\mathrm{CO}_{2}$ assimilation in symbiosis. Four mutants impaired in symbiotic nitrogen fixation were affected in three genes involved in $\mathrm{CO}_{2}$ assimilation via the Calvin Benson Bassham (CBB) cycle (cbbL, BRADO2274; $c b b E$, BRADO2842; and $c b b K$, BRADO1122). Genome annotation predicts that strain ORS278 possesses all the genes required for a functional $\mathrm{CBB}$ cycle.

The key step of the CBB cycle is the carboxylation of ribulose bis phosphate catalyzed by Rubisco (ribulose bisphosphate carboxylase/oxygenase), which is encoded by the $c b b L$ and $c b b S$ genes. Interestingly, as in strain BTAi1, ORS278 has two distantly related Rubisco gene sets ( $c b b L$ and $c b b S ; 57 \%$ amino acid identity between the two CbbL). BRADO1659/ BRADO1660 is found in other rhizobia (including B. japonicum) and is associated with other $c b b$ genes. BRADO2274/ BRADO2275, on the other hand, seems to be restricted to photosynthetic bradyrhizobia. It is located in a genomic island acquired by lateral transfer (Giraud et al. 2007) and is linked to genes involved in a $\mathrm{CO}_{2}$ concentration mechanism (carboxysome encoding genes). The fact that the BRADO1659/ BRADO1660 gene set does not complement the mutation in BRADO2274 strongly suggests that they play different physiological roles.

Other rhizobia (BTAi1, B. japonicum, and S. meliloti) have been reported to fix $\mathrm{CO}_{2}$ via the $\mathrm{CBB}$ cycle under free-living conditions (Hungria et al. 1993; Manian et al. 1984, Pickering and Oresnik 2008) but, to our knowledge, bacterial $\mathrm{CO}_{2}$ fixation has never been reported to be required for effective nitrogen fixation in a rhizobium-legume symbiosis. Our aim is now to determine the exact role of the bacterial CBB cycle during symbiosis. Possibly, photosynthetic bradyrhizobia use the $\mathrm{CBB}$ cycle as an electron sink to equilibrate the red/ox balance or the bacteroids use it as an important carbon supply.

Novel regulatory and signaling proteins involved in bacteroid functioning. A second class of novel symbiosis genes is related to regulation and signaling and is represented by five mutants that exhibit a strong deficiency in their nitrogen fixation activity.

i) BRADO3946 encodes a large sensory histidine kinase of 2,117 amino acids. Interestingly, a previous screening of a mutant library for clones altered in the photosystem synthesis identified mutants in this CDS (Jaubert et al. 2009). It was

Fig. 2. Main metabolic routes found to be important during the symbiosis between ORS278 and Aeschynomene indica. The nodule development-deficient $\left(\mathrm{Ndv}^{-}\right)$mutants are in red underlined, whereas the nitrogen fixation-deficient (Fix $\left.{ }^{-}\right)$mutants are in blue bold. The mutants identified during the previous screening are in green and highlighted with an asterisk (Giraud et al. 2007). For clarity, some biosynthesis pathways are not given in detail but are represented by dotted arrows and enzymatic steps for which symbiotically defective mutants were isolated. R5P = ribose-5-phosphate, G3P = glyceraldehyde-3phosphate, F6P = fructose-6-phosphate, $\mathrm{PBH}=$ polyhydroxybutyrate, $\mathrm{PRPP}=5$-phosphoribosylpyrophosphate, GAR = 5'-phosphoribosylglycinamide, FGAR $=5^{\prime}$-phosphoribosylformylglycinamide, FGAM $=5^{\prime}$-phosphoribosylformylglycinamidine, AIR = 5'-phosphoribosyl-5-aminoimidazole, CAIR = 5'phosphoribosyl-4-carboxy-5-aminoimidazole, SAICAR $=5^{\prime}$-phosphoribosyl-4-(N-succinocarboxamide)-5-aminoimidazole, AICAR = 5'-phosphoribosyl-5amino-4-imidazole carboxamide, FAICAR = 5'-phosphoribosyl-5-formamido-4-imidazole carboxamide, IMP = inosine 5'-monophosphate, XMP = xanthosine 5'-monophosphate, GMP = guanosine 5'-monophosphate, AMP =, adenosine 5'-monophosphate, UMP = uridine 5'-monophosphate, AMP = adenosine $5^{\prime}$-monophosphate, UMP = uridine 5'-monophosphate, $\mathrm{CMP}=$ cytidine-5'-monophosphate, and dTMP $=$ deoxythymidine $55^{\prime}$-monophosphate. 


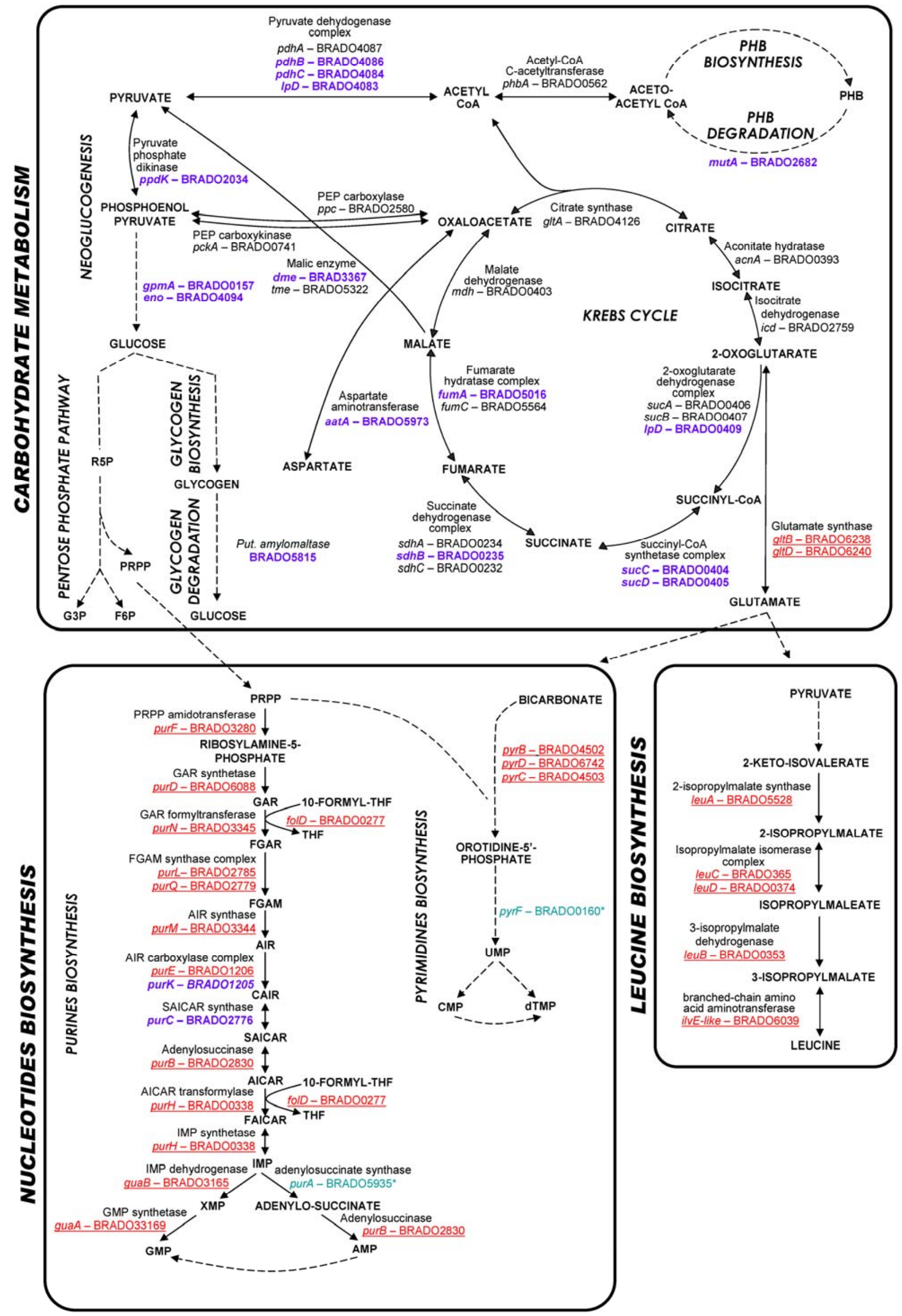

Vol. 23, No. 6, 2010 / 767 
postulated that this gene would control not only photosynthesis but also other metabolic pathways because a homologue was found in the nonphotosynthetic $B$. japonicum USDA110. The drastic nitrogen fixation deficiency of the mutant isolated during this study strengthens the hypothesis that BRADO3946 could act as a global regulator.

ii) BRADO4470 encodes a homologue of LabA (36\% identity and $57 \%$ similarity), identified in cyanobacteria and required for feedback control of KaiC, which is a central component of the circadian clock system (Taniguchi et al. 2007). Although a photoperiodicity is necessary to induce photosystem formation under white light conditions (Giraud and Fleischman 2004), and two kaiBC operons are present in the ORS278 and BTAi1 genomes, circadian gene expression in photosynthetic bradyrhizobia has never been reported. Nevertheless, a circadian control of the nif genes would be particularly beneficial to optimize the symbiotic interaction in the green stem nodules. Indeed, by restricting the synthesis of the nitrogenase to the night time, the deleterious effect of $\mathrm{O}_{2}$ production by the photosynthetic activity of the chloroplasts during the day could be prevented.

iii) BRADO4636 encodes a putative transcriptional regulatory protein related to the MarR family. This CDS is found close to a putative oxidoreductase (BRADO4637) and such genetic organization is well conserved in bacteria, including several rhizobium strains (B. japonicum, S. meliloti, Mesorhizobium loti, and others), suggesting that BRADO4636 could target this oxidoreductase.

iv and v) BRADO3225 and BRADO1477 encode putative chemotaxis genes, a methyl-accepting chemotaxis protein (MCP) and a CheR homologue, respectively. Chemotaxis and motility are known in rhizobia to confer a competitive advantage in the early infection events (Ames and Bergman 1981; Miller et al. 2007). However, once the infection has begun, motility is not required anymore (Caetano-Anollés et al. 1988). Unexpectedly, the mutants in BRADO1477 and BRADO3225 appeared to be drastically impaired in their efficiency to fix $\mathrm{N}_{2}$. It has been shown that some che operons are implicated in controlling cellular functions other than motility, including biofilm formation, initiation of developmental programs, EPS biosynthesis, and so on (Hickman et al. 2005; Kirby and Zusman 2003; Bible et al. 2008). Considering that 4 che operons and $49 \mathrm{MCP}$ are identified in the ORS278 genome, mutants in BRADO1477 and BRADO3225 might be affected in biological processes other than chemotaxis.

Critical role of membrane integrity for efficient nitrogen fixation. Two mutants affected in CDS putatively involved in cellwall biogenesis were isolated: BRADO4549 encodes a putative D-alanyl-D-alanine carboxypeptidase involved in peptidoglycan biosynthesis and BRADO0045 encodes an apolipoprotein $\mathrm{N}$-acyltransferase that transfers a fatty acyl group on membrane lipoproteins. Possibly, BRADO0045 and BRADO4549 may be needed for membrane integrity, which is likely to be critically important during the early stages of bacterial differentiation within plant cells.

\section{Conclusions and perspectives.}

From this study, we gained insights into the metabolism of photosynthetic bradyrhizobia during root symbiosis with Aeschynomene spp. The main routes (TCA cycle, gluconeogenesis, $\mathrm{PHB}$, and glycogen degradation) already known to play a central role in bacteroid metabolism were revealed to be also required by photosynthetic bradyrhizobia for an efficient symbiosis. Furthermore, we identified unexpected biological processes, such as $\mathrm{CO}_{2}$ fixation and chemotaxis, as well as a large number of novel genes, including regulators, which appear to be important for symbiosis. Some of these determinants are conserved among rhizobia, suggesting that they could also be required in other rhizobium-legume symbioses. Further investigations on these newly identified genes are needed to confirm their role during nodulation and, if confirmed, to unravel their precise function in the symbiotic process.

To better characterize the molecular mechanisms involved during the early steps of this Nod-independent symbiotic process, other approaches besides the screening of additional mutants are required, mainly because of the likely redundancy of functions involved in these steps. Therefore, we are currently developing i) a positive screen by transferring a cosmid library of ORS278 in a conventional Rhizobium sp. and selecting transconjugants that elicit nodules or nodule-like structures on A. indica and ii) transcriptomic and proteomic analyses in the presence or absence of root exudates.

\section{MATERIALS AND METHODS}

\section{Bacterial strains and growth conditions.}

Escherichia coli strains were grown at $37^{\circ} \mathrm{C}$ in Luria-Bertani medium (Sambrook et al. 1989). The WT strain Bradyrhizobium ORS278 and its derivatives were cultivated at $37^{\circ} \mathrm{C}$ in modified yeast extract mannitol (YMm) medium (Giraud et al. $2000)$ or in MM. The MM (pH 6.8) contained $\mathrm{K}_{2} \mathrm{HPO}_{4}(1.6 \mathrm{~g}$ liter $\left.{ }^{-1}\right), \mathrm{KH}_{2} \mathrm{PO}_{4}\left(0.8 \mathrm{~g} \mathrm{liter}^{-1}\right)$, sodium glutamate $\left(0.56 \mathrm{~g} \mathrm{liter}^{-1}\right)$, xylose $\left(3 \mathrm{~g} \mathrm{liter}^{-1}\right), \mathrm{NaCl}\left(50 \mathrm{mg} \mathrm{liter}^{-1}\right), \mathrm{MgSO}_{4}\left(50 \mathrm{mg} \mathrm{liter}^{-1}\right)$, $\mathrm{CaCl}_{2}\left(40 \mathrm{mg} \mathrm{liter}^{-1}\right), \mathrm{ZnSO}_{4} \cdot 7 \mathrm{H}_{2} \mathrm{O}\left(22 \mathrm{mg} \mathrm{liter}^{-1}\right), \mathrm{H}_{3} \mathrm{BO}_{3}(11$ mg liter $\left.{ }^{-1}\right), \mathrm{MnCl}_{2} \cdot 4 \mathrm{H}_{2} \mathrm{O}\left(5 \mathrm{mg} \mathrm{liter}^{-1}\right), \mathrm{FeSO}_{4} \cdot 7 \mathrm{H}_{2} \mathrm{O}(5 \mathrm{mg}$ liter $\left.{ }^{-1}\right), \mathrm{CoCl}_{2} \cdot 6 \mathrm{H}_{2} \mathrm{O}\left(1.6 \mathrm{mg} \mathrm{liter}^{-1}\right), \mathrm{CuSO}_{4} \cdot 5 \mathrm{H}_{2} \mathrm{O}(1.6 \mathrm{mg}$ liter $\left.{ }^{-1}\right),\left(\mathrm{NH}_{4}\right)_{6} \mathrm{Mo}_{7} \mathrm{O}_{24} \cdot 4 \mathrm{H}_{2} \mathrm{O}$ (1.1 $\left.\mathrm{mg} \mathrm{liter}^{-1}\right), \mathrm{Na}_{4}$ EDTA . $4 \mathrm{H}_{2} \mathrm{O}\left(60 \mathrm{mg} \mathrm{liter}^{-1}\right)$, riboflavin $\left(200 \mu \mathrm{g}\right.$ liter $\left.^{-1}\right)$, biotin $(120 \mu \mathrm{g}$ liter $\left.^{-1}\right)$, thiamine-HCl $\left(800 \mu \mathrm{g}\right.$ liter $\left.^{-1}\right)$, myo-inositol $(48 \mu \mathrm{g}$ liter $\left.^{-1}\right), \mathrm{p}$-aminobenzoic acid $\left(80 \mu \mathrm{g} \mathrm{liter}^{-1}\right)$, nicotinic acid $(500$ $\mu \mathrm{g}$ liter $\left.^{-1}\right)$, calcium panthotenate $\left(800 \mu \mathrm{g} \mathrm{liter}^{-1}\right)$, and cyanocobalamin $\left(1 \mu \mathrm{g}\right.$ liter $\left.^{-1}\right)$. When required, the media were supplemented with kanamycin $(50 \mu \mathrm{g} / \mathrm{ml})$ for $E$. coli and with a mixture of kanamycin $(50 \mu \mathrm{g} / \mathrm{ml})$ and nalidixic acid $(35 \mu \mathrm{g}$ liter $^{-1}$ ) for ORS278 mutants.

\section{Transposon mutagenesis and colony selection.}

Transposon insertions in the ORS278 genome were generated by a biparental mating with Bradyrhizobium sp. strain ORS278 and E. coli sp. strain BW20767 containing the plasmid pCRS487 harboring the minitransposon mTn5-GNm (Reeve et al. 1999) according to the protocol previously described (Jaubert et al. 2009). The clones were picked and arrayed into 96-well plates, each plates containing $120 \mu \mathrm{l}$ of YMm medium with selective antibiotics. Plates were incubated for 7 days at $37^{\circ} \mathrm{C}$ under agitation $(100 \mathrm{rpm})$. After addition of glycerol $(50 \%)$ at $40 \mu \mathrm{l} /$ well, the plates were stored at $-80^{\circ} \mathrm{C}$.

\section{In planta screening.}

A. indica seed were surface sterilized with concentrated (96\%) sulfuric acid for $30 \mathrm{~min}$, abundantly rinsed with sterile water, and soaked overnight in sterile water. The seed were transferred onto sterile $0.8 \%$ water agar plates and germinated upside-down overnight at $37^{\circ} \mathrm{C}$ in the dark. One-day-old seedlings were transferred to 48 -well plates $(6.0-\mathrm{ml}$ storage plate, ref: AB-0988; Thermoscientific) previously filled with $5.6 \mathrm{ml}$ of nitrogen-free Jensen's medium (Vincent 1970), inoculated individually with $100 \mu \mathrm{l}$ of 7-day-old mutant cultures, and covered by sterile adhesive aluminum foil. To allow passage of the radicle, a small hole in the aluminum cover was made using a sterile toothpick. The 48-well plates containing the seedlings were placed in a small greenhouse containing 1 liter of sterile water and placed for 3 weeks in a $28^{\circ} \mathrm{C}$ growth chamber with a 16-h light regime and $70 \%$ humidity. Mutants impaired in their 
ability to form nodules $\left(\mathrm{Ndv}^{-}\right)$or to fix nitrogen by eliciting yellow nodules $\left(\mathrm{Fix}^{-}\right)$when tested on single plants were then reinoculated onto two plants each. Symbiotic phenotypes were determined as above and the symbiotically defective mutants were reinoculated onto six plants each and their phenotype was reexamined as above. Mutants showing a symbiotic deficiency and for which the Tn5 insertion site determination was successful were retained for further characterization.

\section{Nitrogenase activity.}

Acetylene reduction assays were performed 3 weeks after inoculation. Two whole plants were placed into $125-\mathrm{ml}$ glass vials sealed with rubber septa. Air $(12.5 \mathrm{ml})$ was removed from each vial, to which $12.5 \mathrm{ml}$ of acetylene was then injected. Gas samples $(1 \mathrm{ml})$ were withdrawn after $3 \mathrm{~h}$ of incubation at $25^{\circ} \mathrm{C}$ and the ethylene produced was measured by gas chromatography. The assay was done in triplicate and compared with plants that had been inoculated with the WT strain.

\section{Bacterial growth rate.}

Cultures grown for 5 days in YMm medium were pelleted by centrifugation, washed twice with $\mathrm{NaCl}\left(9 \mathrm{~g} \mathrm{liter}^{-1}\right)$ and inoculated in MM or YMm medium to obtain an initial optical density at $600 \mathrm{~nm}\left(\mathrm{OD}_{600}\right)$ of 0.02 . After 4 days of incubation at $37^{\circ} \mathrm{C}$, the $\mathrm{OD}_{600}$ of each culture was measured and compared with the WT strain.

\section{Transposon insertion location.}

Transposon insertion location was determined by a twostage semidegenerative PCR and sequencing protocol according to Jacobs and associates (2003). The precise location of the Tn5 insertions in the ORS278 genome was determined via a BLASTN search using the obtained sequences as query.

\section{ACKNOWLEDGMENTS}

This work was supported by grants from the French national research agency (ANR-06-NLAN-0095) and the "Ministère de l'Education Nationale, de 1'Enseignement Supérieur et de la Recherche" (fellowship to K. Bonaldi) and the European Molecular Biology Organization (fellowship to B. Gourion). We thank A. Verméglio (CEA, Cadarache, France) for critical reading of the manuscript, D. Gourion (Department of Mathematics, University of Avignon, France) for stimulating discussions, and our anonymous reviewers for their useful comments on the manuscript.

\section{LITERATURE CITED}

Amadou, C., Pascal, G., Mangenot, S., Glew, M., Bontemps, C., Capela, D., Carrère, S., Cruveiller, S., Dossat, C., Lajus, A., Marchetti, M., Poinsot, V., Rouy, Z., Servin, B., Saad, M., Schenowitz, C., Barbe, V., Batut, J., Médigue, C., and Masson-Boivin, C. 2008. Genome sequence of the beta-rhizobium Cupriavidus taiwanensis and comparative genomics of rhizobia. Genome Res. 18:1472-1483.

Ames, P., and Bergman, K. 1981. Competitive advantage provided by bacterial motility in the formation of nodules by Rhizobium meliloti. J. Bacteriol. 148:728-729.

Arora, N. 1954. Morphological development of the root and stem nodules of Aeschynomene indica L. Phytomorphology 4:211-216.

Barnett, M. J., Fisher, R. F., Jones, T., Komp, C., Abola, A. P., BarloyHubler, F., Bowser, L., Capela, D., Galibert, F., Gouzy, J., Gurjal, M., Hong, A., Huizar, L., Hyman, R. W., Kahn, D., Kahn, M. L., Kalman, S., Keating, D. H., Palm, C., Peck, M. C., Surzycki, R., Wells, D. H., Yeh, K. C., Davis, R. W., Federspiel, N. A., and Long, S. R. 2001. Nucleotide sequence and predicted functions of the entire Sinorhizobium meliloti pSymA megaplasmid. Proc. Natl. Acad. Sci. U.S.A. 98:98839888.

Bible, A. N., Stephens, B. B., Ortega, D. R., Xie, Z., and Alexandre, G. 2008. Function of a chemotaxis-like signal transduction pathway in modulating motility, cell clumping, and cell length in the alphaproteobacterium Azospirillum brasilense. J. Bacteriol. 190:6365-6375.

Boyd, L. A., Woytowich, A., and Selvaraj, G. 1993. Target sequence specificity of transposon Tn 5 in the absence of major hotspots in the plasmid
pBR322: Identification of a new hotspot. Plasmid 30:155-158.

Caetano-Anollés, G., Wall, L. G, Micheli, A. T. D., Macchi, A. M., Bauer, W. D, and Favelukes, G. 1988. Role of motility and chemotaxis in efficiency of nodulation by Rhizobium meliloti. Plant Physiol. 86:12281235 .

Chandler, M. R. 1978. Some observations on infection of Arachis hypogaea L. by Rhizobium. J. Exp. Bot. 29:749-755.

Chandler, M. R., Date, R. A., and Roughley, R. J. 1982. Infection and rootnodule development in Stylosanthes species by Rhizobium. J. Exp. Bot. 33:47-57.

Charles, T. C., and Aneja, P. 1999. Methylmalonyl-CoA mutase encoding gene of Sinorhizobium meliloti. Gene 226:121-127.

Dénarié, J., and Bergeron, B. 1976. Effects of some mutations on symbiotic properties of Rhizobium. Pages 47-61 in: Symbiotic Nitrogen Fixation. P. S. Nutman, ed. Cambridge University Press, Cambridge.

Fischer, H. M. 1994. Genetic regulation of nitrogen fixation in rhizobia. Microbiol. Rev. 58:352-386.

Frugier, F., Kosuta, S., Murray, J. D., Crespi, M., and Szczyglowski, K. 2008. Cytokinin: Secret agent of symbiosis. Trends Plant Sci. 13:115120.

Geurts, R., Fedorova, E., and Bisseling, T. 2005. Nod factor signaling genes and their function in the early stages of Rhizobium infection. Curr. Opin. Plant Biol. 8:346-52.

Gibson, K. E., Kobayashi, H., and Walker, G.C. 2008. Molecular determinants of a symbiotic chronic infection. Annu. Rev. Genet. 42:413-441.

Giraud, E., and Fleischman, D. 2004. Nitrogen-fixing symbiosis between photosynthetic bacteria and legumes. Photosynth. Res. 82:115-130.

Giraud, E., Hannibal, L., Fardoux, J., Verméglio, A., and Dreyfus, B. 2000. Effect of Bradyrhizobium photosynthesis on stem nodulation of Aeschynomene sensitiva. Proc. Natl. Acad. Sci. U.S.A. 97:14795-14800.

Giraud, E., Moulin, L., Vallenet, D., Barbe, V., Cytryn, E., Avarre, J. C., Jaubert, M., Simon, D., Cartieaux, F., Prin, Y., Bena, G., Hannibal, L., Fardoux, J., Kojadinovic, M., Vuillet, L., Lajus, A., Cruveiller, S., Rouy, Z., Mangenot, S., Segurens, B., Dossat, C., Franck, W. L., Chang, W. S., Saunders, E., Bruce, D., Richardson, P., Normand, P., Dreyfus, B. Pignol, D., Stacey, G., Emerich, D., Verméglio, A., Médigue, C., and Sadowsky, M. 2007. Legumes symbioses: Absence of Nod genes in photosynthetic bradyrhizobia. Science 316:1307-1312.

Gonzalez-Rizzo, S., Crespi, M., and Frugier F. 2006. The Medicago truncatula CRE1 cytokinin receptor regulates lateral root development and early symbiotic interaction with Sinorhizobium meliloti. Plant Cell 18:2680-2693.

Goormachtig, S., Capoen, W., and Holsters, M. 2004. Rhizobium infection: Lessons from the versatile nodulation behaviour of water-tolerant legumes. Trends Plant Sci. 9:518-522.

Gourion, B., Sulser, S., Frunzke, J., Francez-Charlot, A., Stiefel, P., Pessi, G., Vorholt, J. A., and Fischer, H. M. 2009. The PhyR-sigma(EcfG) signalling cascade is involved in stress response and symbiotic efficiency in Bradyrhizobium japonicum. Mol Microbiol. 73:291-305.

Hickman, J. W., Tifrea, D. F., and Harwood, C. S. 2005. A chemosensory system that regulates biofilm formation through modulation of cyclic diguanylate levels. Proc. Natl. Acad. Sci. U.S.A. 102:14422-14427.

Hungria, M., Ellis, J. M., Hardy, R. W. F., and Eaglesham, A. R. J. 1993. Light-stimulated $\mathrm{CO}_{2}-\mathrm{C}-14$ uptake and acetylene-reduction by bacteriochlorophyll containing stem nodule isolate BTAi-1. Biol. Fertil. Soils 15:208-214.

Jacobs, M. A, Alwood, A., Thaipisuttikul, I., Spencer, D., Haugen, E., Ernst, S., Will. O., Kaul, R., Raymond. C., Levy, R., Chun-Rong, L., Guenthner, D., Bovee, D., Olson, M. V., and Manoil, C. 2003. Comprehensive transposon mutant library of Pseudomonas aeruginosa. Proc. Natl. Acad. Sci. U.S.A. 100:14339-14344.

Jaubert, M., Hannibal, L., Fardoux, J., Giraud, E., and Verméglio, A. 2009. Identification of novel genes putatively involved in the photosystem synthesis of Bradyrhizobium sp. ORS 278. Photosynth. Res. 100:97-105.

Jones, K. M., Kobayashi, H., Davies, B. W., Taga, M. E., and Walker, G. C. 2007. How rhizobial symbionts invade plants: The SinorhizobiumMedicago model. Nat. Rev. Microbiol. 5:619-633.

Kaneko, T., Nakamura, Y., Sato, S., Minamisawa, K., Uchiumi, T. Sasamoto, S., Watanabe, A., Idesawa, K., Iriguchi, M., Kawashima, K., Kohara, M., Matsumoto, M., Shimpo, S., Tsuruoka, H., Wada, T., Yamada, M., and Tabata, S. 2002. Complete Genomic sequence of nitrogen-fixing symbiotic bacterium Bradyrhizobium japonicum USDA110. DNA Res. 9:189-197.

Kirby, J. R., and Zusman, D. R. 2003. Chemosensory regulation of developmental gene expression in Myxococcus xanthus. Proc. Natl. Acad. Sci. U.S.A. 100:2008-2013.

Lodwig, E. M., Leonard, M., Marroqui, S., Wheeler, T. R., Findlay, K., Downie, J. A., and Poole, P. S. 2005. Role of polyhydroxybutyrate and glycogen as carbon storage compounds in pea and bean bacteroids. Mol. Plant-Microbe Interact. 18:67-74. 
Long, S. R. 1989. Rhizobium genetics. Annu. Rev. Genet. 23:483-506.

Manian, S. S., Gumbleton, R., Buckley, A. M., and O'Gara, F. 1984. Nitrogen fixation and carbon dioxide assimilation in Rhizobium japonicum. Appl. Environ. Microbiol. 48:276-279.

Martin, G. B., Chapman, K. A., and Chelm, B. K. 1988. Role of the Bradyrhizobium japonicum ntrC gene product in differential regulation of the glutamine synthetase II gene $(g \ln I I)$. J. Bacteriol. 170:5452-5459.

Miller, L. D., Yost, C. K., Hynes, M. F., and Alexandre, G. 2007. The major chemotaxis gene cluster of Rhizobium leguminosarum bv. viciae is essential for competitive nodulation. Mol. Microbiol. 63:348-62.

Moulin, L., Munive, A., Dreyfus, B., and Boivin-Masson, C. 2001. Nodulation of legumes by members of the beta-subclass of Proteobacteria. Nature 411:948-950

Murray, J. D., Karas, B. J., Sato, S., Tabata, S., Amyot, L., and Szczyglowski K. 2007. A cytokinin perception mutant colonized by Rhizobium in the absence of nodule organogenesis. Science 315:101104

Newman, J. D., Diebold, R. J., Schultz, B. W., and Noel, K. D. 1994. Infection of soybean and pea nodules by Rhizobium spp. purine auxotrophs in the presence of 5-aminoimidazole-4-carboxamide riboside. J. Bacteriol. 176:3286-3294

Noel, K. D., Diebold, R. J., Cava, J. R., and Brink, B. A. 1988. Rhizobial purine and pyrimidine auxotrophs: Nutrient supplementation, genetic analysis, and the symbiotic requirement for de novo purine biosynthesis. Arch. Microbiol. 149:499-506.

Oldroyd, G. E. 2007. Plant science. Nodules and hormones. Science 315:52-53

Oldroyd, G. E., and Downie, J. A. 2008. Coordinating nodule morphogenesis with rhizobial infection in legumes. Annu. Rev. Plant Biol. 59:519-46.

Pain, A. N. 1979. Symbiotic properties of antibiotic-resistant and auxotrophic mutants of Rhizobium leguminosarum. J. Appl. Bacteriol. 47:53-64

Perret, X., Staehelin, C., and Broughton, W. J. 2000. Molecular basis of symbiotic promiscuity. Microbiol. Mol. Biol. Rev. 64:180-201.

Pickering, B. S., and Oresnik, I. J. 2008. Formate-dependent autotrophic growth in Sinorhizobium meliloti. J. Bacteriol. 190:6409-6418.

Reeve, W. G., Tiwari, R. P., Worsley, P. S., Dilworth, M. J., Glenn, A. R., and Howieson, J. G. 1999. Constructs for insertional mutagenesis, transcriptional signal localization and gene regulation studies in root nodule and other bacteria. Microbiology 145:1307-1316.

Sambrook, J., Fritsch, E. F., Maniatis, T., and Irwin, N. 1989. Molecular Cloning: A Laboratory Manual, $2 \mathrm{n}$ ed. Cold Spring Harbor Laboratory Press, Plainview, NY, U.S.A.
Sprent, J. I. 2007. Evolving ideas of legume evolution and diversity: A taxonomic perspective on the occurrence of nodulation. New Phytol. 174:11-25.

Stacey, G., Libault, M., Brechenmacher, L., Wan, J., and May, G. D. 2006 Genetics and functional genomics of legume nodulation. Curr. Opin. Plant Biol. 9:110-121.

Szeto, W. W., Nixon, B. T., Ronson, C. W., and Ausubel, F. M. 1987. Identification and characterization of the Rhizobium meliloti ntrC gene: $R$. meliloti has separate regulatory pathways for activation of nitrogen fixation genes in free-living and symbiotic cells. J. Bacteriol. 169:1423 1432.

Taniguchi, Y., Katayama, M., Ito, R., Takai, N., Kondo, T., and Oyama, T. 2007. LabA: A novel gene required for negative feedback regulation of the cyanobacterial circadian clock protein KaiC. Genes Dev. 21:60-70.

Tirichine, L., Sandal, N., Madsen, L. H., Radutoiu, S., Albrektsen, A. S, Sato, S., Asamizu, E., Tabata, S., and Stougaard, J. 2007. A gain-offunction mutation in a cytokinin receptor triggers spontaneous root nodule organogenesis. Science 315:104-107.

Trainer, M. A., and Charles, T. C. 2006. The role of PHB metabolism in the symbiosis of rhizobia with legumes. Appl. Microbiol. Biotechnol 71:377-386.

Vallenet, D., Labarre, L., Rouy, Z., Barbe, V., Bocs, S., Cruveiller, S., Lajus, A., Pascal, G., Scarpelli, C., and Médigue, C. 2006. MaGe: A microbial genome annotation system supported by synteny results. Nucleic Acids Res. 34:53-65.

Vincent, J. M. 1970. A Manual for the Practical Study of Root-Nodule Bacteria. IBP Handbook 15. International Biological Programme. Blackwell Scientific Publications, Oxford.

Young, J. P., Crossman, L. C., Johnston, A. W., Thomson, N. R., Ghazoui, Z. F., Hull K. H., Wexler, M., Curson, A. R., Todd, J. D., Poole, P. S., Mauchline, T. H., East, A. K., Quail, M. A., Churcher, C., Arrowsmith, C., Cherevach, I., Chillingworth, T., Clarke, K., Cronin, A., Davis, P., Fraser, A., Hance, Z., Hauser, H., Jagels, K., Moule, S., Mungall, K., Norbertczak, H., Rabbinowitsch, E., Sanders, M., Simmonds, M., Whitehead, S., and Parkhill, J. 2006. The genome of Rhizobium leguminosarum has recognizable core and accessory components. Genome Biol. 7:R34.

\section{AUTHOR-RECOMMENDED INTERNET RESOURCE}

GenoScope MaGe NewNodBrady database: www.genoscope.cns.fr/agc/newnodbrady 